\title{
Lieb-Mattis ferrimagnetism in diluted magnetic semiconductors
}

\author{
R.O. Kuzian, ${ }^{1,2}$ J. Richter, ${ }^{3}$ M. D. Kuz'min,${ }^{4}$ and R. Hayn ${ }^{4}$ \\ ${ }^{1}$ Institute for Problems of Materials Science NASU, Krzhizhanovskogo 3, 03180 Kiev, Ukraine \\ ${ }^{2}$ Donostia International Physics Center (DIPC), ES-20018 Donostia-SanSebastian, Spain \\ ${ }^{3}$ Institut für Theoretische Physik, Otto-von-Guericke-Universität Magdeburg, \\ PF 4120, D - 39016 Magdeburg, Germany \\ ${ }^{4}$ Aix-Marseille Université, IM2NP-CNRS UMR 7334, \\ Campus St. Jérôme, Case 142, 13397 Marseille, France
}

(Dated: 11.10.15)

\begin{abstract}
We show the possibility of long-range ferrimagnetic ordering with a saturation magnetisation of $\sim 1 \mu_{B}$ per spin for arbitrarily low concentration of magnetic impurities in semiconductors, provided that the impurities form a superstructure satisfying the conditions of the Lieb-Mattis theorem. Explicit examples of such superstructures are given for the wurtzite lattice, and the temperature of ferrimagnetic transition is estimated from a high-temperature expansion. Exact diagonalization studies show that small fragments of the structure exhibit enhanced magnetic response and isotropic superparamagnetism at low temperatures. A quantum transition in a high magnetic field is considered and similar superstructures in cubic semiconductors are discussed as well.

PACS numbers: 75.10.-b, 75.20.-g, 75.50.Gg, 75.50.Pp
\end{abstract}

In order to launch the engineering of a new generation of electronic devices, one needs new materials with special properties. For instance, spintronics has a need for room-temperature ferromagnetic semiconductors ${ }^{1}$. Since the discovery of high- $T_{C}$ ferromagnetism in GaAs: $\mathrm{Mn}^{2}$ and the prediction of room-temperature ferromagnetism in $p$-doped $\mathrm{ZnO}: \mathrm{Co}, \mathrm{Mn}$ systems ${ }^{3}$, a lot of attempts have been made to obtain ferromagnetism in transition metal doped $\mathrm{ZnO}$, GaN and in other oxides and nitrides. The $p$-type carriers doping is necessary for the $p$ - $d$ Zener ferromagnetic long-range interaction? Up to now all attempts to obtain $\mathrm{ZnO}$ with $p$-type current carriers have failed. Nevertheless, several reports of "ferromagnetic" room temperature behavior have been published 5 5! 7 . "Perhaps the most surprising development of the past decade in the science of magnetic materials is the abundant observations of spontaneous magnetization persisting to above room temperature in semiconductors and oxides, in which no ferromagnetism was expected at any temperature, particularly in the $p$ - $d$ Zener model ${ }^{p[5}$.

In the absence of $p$-type current carriers, the interaction between magnetic impurities is governed by the superexchange mechanism. Superexchange is often regarded as an obstacle in the way towards magnetic semiconductors as it has antiferromagnetic (AFM) character and tends to anti-align the interacting spins, leading to a cancellation of the net magnetization. In fact, the AFM interaction does not preclude spontaneous magnetization. In a seminal paper ${ }^{8}$, E. Lieb and D. Mattis showed that the ground state of an AFM system depends on the topology of the interacting bonds and, under certain conditions, it is ferrimagnetic rather than AFM. The Lieb-Mattis theorem applies if there is no magnetic frustration in the spin system.

In this communication we study various structures formed by the interacting magnetic impurities in wurtzite semiconductors. We take antiferromagnetic nearest neighbor interaction into account and consider diluted lattices without frustration, in order to remain within the Lieb-Mattis scheme. First we construct several finite clusters that show an enhanced magnetic response at low temperatures. Not alone do they possess a net magnetic moment, they all share a further interesting peculiarity: below a certain temperature their magnetic susceptibility exceeds that of non-interacting spins. We call it isotropic superparamagnetic response ${ }^{910}$. Next we construct extended lattices of these clusters, which undergo a ferrimagnetic ordering transition at a finite temperature. The average ground-state spin per magnetic ion of spin $S$ tends to a finite value (of about $S / 3$ ) despite the low concentration of magnetic ions. The extension of our idea to other lattices and the influence of frustration will be briefly discussed at the end of the communication.

We take the interaction in the form

$$
\hat{H}=\frac{1}{2} \sum_{\mathbf{R}, \mathbf{r}} J_{\mathbf{r}} \hat{\mathbf{S}}_{\mathbf{R}} \hat{\mathbf{S}}_{\mathbf{R}+\mathbf{r}},
$$

i.e., we adopt the notation $J_{\mathbf{r}}$ for the interaction between one pair of spins 11 . We assume that only the nearest-neighbor (in the metal sublattice) interaction is nonzero. This assumption is relevant to magnetic semiconductors, where the nearest-neighbor exchange dominates 12 . Two kinds of nearest neighborships are present in wurtzites: those where both ions lie in the same plane and those where they lie in two adjacent planes. The corresponding exchange integrals, $J_{1}$ (inplane) and $J_{2}$ (out-of-plane), are different ${ }^{15}[17$.

The magnetic response of a system is characterized by its magnetic susceptibility. Talking about a compound $\mathrm{A}_{1-x} \mathrm{M}_{x} \mathrm{X}$ (where $\mathrm{X}$ is a ligand of $\mathrm{V}$ or $\mathrm{VI}$ group, $\mathrm{A}$ is a metal of IIId or IId group, and $\mathrm{M}$ is a transition metal), we shall attribute all the magnetic moment to transition metal ions (TMIs) only. We now introduce the magnetic 
susceptibility per one spin,

$$
\chi \equiv \frac{\mu_{M}}{H},
$$

where $\mu_{M}$ is the average magnetic moment of one TMI. For non-interacting spins, the susceptibility obeys the Curie law $\chi_{C}=\left[\left(g \mu_{B}\right)^{2} S(S+1)\right] /\left(3 k_{B} T\right)$, where $S$ is the spin of the TMI and $g$ is its gyromagnetic ratio. Besides isolated spins, TMI impurities may form pairs, trimers, tetramers, and more complex structures (see Fig. 1). The
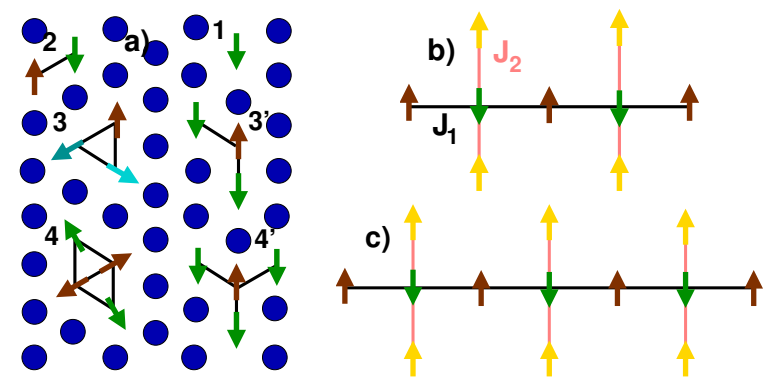

FIG. 1. (Color online) a) : Complexes formed by transition metal impurities (arrows): isolated ions (1), dimers (2), trimers $\left(3,3^{\prime}\right)$, tetramers $\left(4,4^{\prime}\right)$. Black solid line segments depict the nearest-neighbor interaction $J_{1}$ bonds. One wurtzite $a b$ plane is shown, blue circles denote non-magnetic host metal ions, ligands are not shown. b), c) : More complex LiebMattis systems with ferrimagnetic ground state: linear chains of impurities in the $a b$ plane "decorated" by spins in adjacent planes (gold arrows); pink line segments depict $J_{2}$ bonds.

antiferromagneitc interaction depresses the magnetic response at high temperatures. For $T \gg J_{\max } S(S+1) \equiv$ $T_{s}$, the susceptibility of an interacting system obeys the Curie-Weiss law $\chi_{C W}=\left[\left(g \mu_{B}\right)^{2} S(S+1)\right] /\left[3 k_{B}(T-\theta)\right]<$ $\chi_{C}$, with $-\theta=[S(S+1)] /\left(3 k_{B} N\right) \sum_{\mathbf{R}, \mathbf{r}(\mathbf{R})} J_{\mathbf{r}(\mathbf{R})}$. Here $N$ is the number of spins and $J_{\max }$ is the strongest exchange interaction in the system, $\mathbf{R}$ runs over all spins of the lattice, and $\mathbf{r}$ runs over all nearest neighbors of each spin.

At temperatures $T \lesssim T_{s}$, the response of the system depends on its geometry. Analytic expressions for the susceptibility can be obtained for small systems $\frac{18}{18}$. Fig. 22 shows the results for the simplest $S=1 / 2$ case. We see that at $T \sim T_{s}$ the response of three spins arranged linearly $\mathbf{3}^{\prime}$ is larger than that of a triangular arrangement of the same spins 3 . For 4 -spin systems we see the striking difference between the response of a star arrangement $\mathbf{4}^{\prime}$ and that of a rhombus 4.

Even more interesting is the response of the complexes shown in Fig. 1p,c. Each one of these systems can be decomposed into two sublattices A and B (denoted by arrows "up" and "down"), the interaction being nonzero only between sites that belong to different sublattices. Such a system satisfies the requirements of the LiebMattis theorem 8 , and possesses a ferrimagnetic ground state with total spin $S_{g}=S\left|N_{A}-N_{B}\right|$. In this case, the term "ferrimagnetic" refers to correlations of the spins
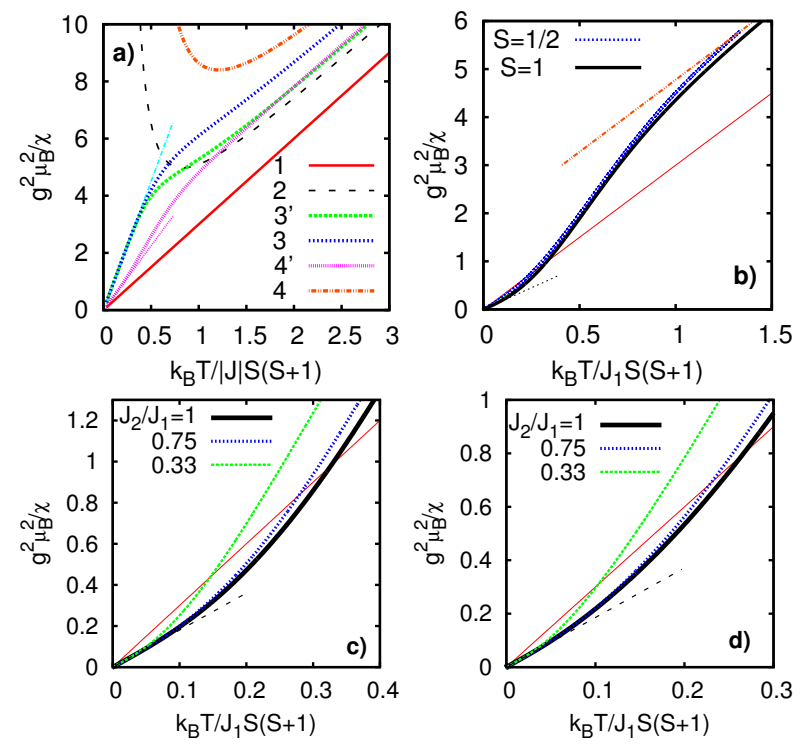

FIG. 2. (Color online) Inverse susceptibility (per spin) $\chi^{-1}$ for the complexes shown in Fig. 1. Straight solid red line shows the Curie law $\chi_{C}^{-1}$; straight dashed lines show the lowtemperature asymptotics: "super" -paramagnetic Curie laws $\left(g \mu_{B}\right)^{2} / \chi_{g}=3 N k_{B} T /\left[S_{g}\left(S_{g}+1\right)\right]$ for Lieb-Mattis systems. a) : clusters shown in Fig. 1 a with $S=1 / 2$; b) : the complex shown in Fig. $1 \mathrm{p}$ with two different values of spin $S$; the straight dash-dotted red line is the high- $T$ Curie-Weiss asymptote. c) the same complex with $S=1$ and various values of $\left.J_{2} / J_{1} ; \mathbf{d}\right)$ the complex shown in Fig. 1 with $S=1 / 2$ and various values of $J_{2} / J_{1}$.

in the ground state, in the absence of a long-range magnetic order ${ }^{[19}$. We have performed full exact diagonalization studies (ED) of thermodynamic properties of clusters shown in Fig. 1b,c using J. Schulenburg's spinpack program 20121 . The susceptibility $\chi(T)$ is calculated as the ratio of the induced magnetization $M$ to the "vanishing" magnetic field $H=10^{-5} J_{1} / g \mu_{B}$. One observes in Figure 2 b,c,d that the response of the systems shown in Fig. 1 $\mathrm{p}, \mathrm{c}$ exceeds the response of non-interacting spins at low temperature. Thus, an antiferromagnetic interaction may result in an enhancement of magnetic response if the geometry of spin arrangement favors the formation of a ferrimagnetic ground state. Then for temperatures $T \ll T_{s}$ the susceptibility per spin shows superparamagnetic response $\chi_{g}=\left[\left(g \mu_{B}\right)^{2} S_{g}\left(S_{g}+1\right)\right] /\left[3 k_{B} T\left(N_{A}+N_{B}\right)\right]$. Evidently, the enhancement of the low-temperature response takes place, if

$$
K \equiv \frac{\chi_{g}}{\chi_{C}}=\frac{\left|N_{A}-N_{B}\right|\left(\left|N_{A}-N_{B}\right| S+1\right)}{\left(N_{A}+N_{B}\right)(S+1)}>1
$$

Not every system satisfying the requirements of the LiebMattis theorem and having a ferrimagnetic ground state has an enhanced susceptibility. Thus, the clusters $3^{\prime}$ $\left(N_{A}=1, N_{B}=2\right)$ and $4^{\prime}\left(N_{A}=1, N_{B}=3\right)$ both have $K<1$, i.e. their response is weaker than that of the same number of non-interacting spins.

The " $\mathrm{S}$ "-shape form of the $T$-dependence of the inverse 
susceptibility (Figure 20) was previously reported for small fragments of ferrimagnetic superstructure in double perovskites 1022 . It interpolates between the Curie-Weiss law $\chi_{C W}$ at $T \gg T_{s}$, and the "super"-spin Curie law $\chi_{g}=K \chi_{C}$ at $T \ll T_{s}$.

If impurity spins arrange themselves in a periodic superstructure having two (or more) non-equivalent spin positions, a ferrimagnetic ground state is possible for this superstructure. Let us denote the number of spins in the superstructure unit cell $n_{A}+n_{B}$, where A and B refer to the non-equivalent positions. If the spins of the sublattice A interact (antiferromagnetically) only with the spins of the sublattice $\mathrm{B}$ (absence of frustration), and $n_{A} \neq n_{B}$, the ground-state spin of the unit cell is $S_{c}=S\left|n_{A}-n_{B}\right|^{[8}$. For a fragment of such a ferrimagnetic superstructure

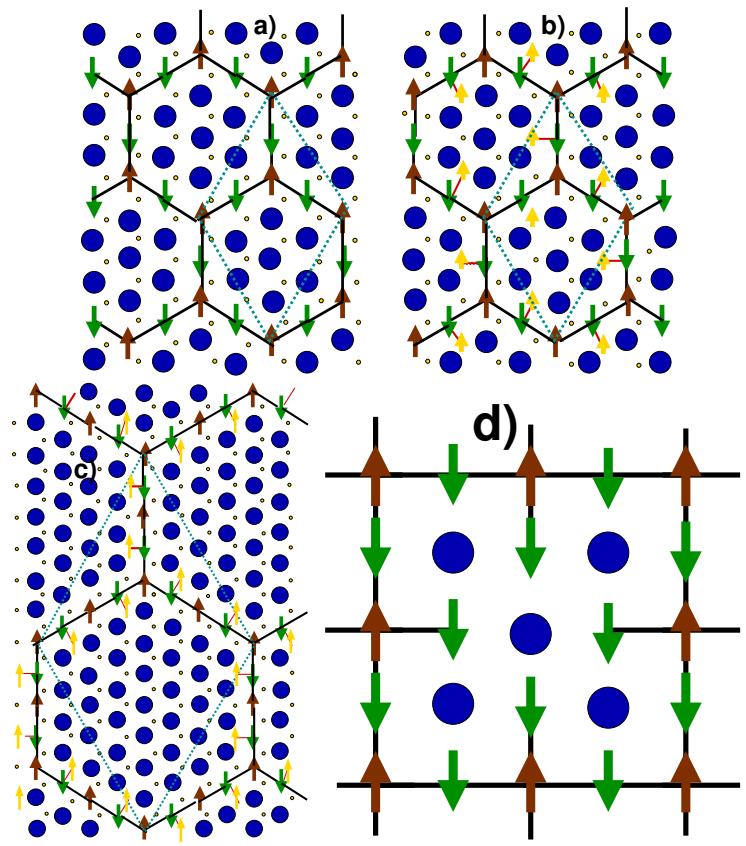

FIG. 3. (Color online) Examples of ferrimagnetic superstructures a), b) : flat and three-dimensional two-leg honeycombs, $L=1$; c) : four-leg honeycomb, $L=2$; d) : a unit cell of a square network, it may be also regarded as a face of cubic unit cell. The notations is the same as in Fig. 1] The cyan rhombi show the unit cells.

containing $N_{c}$ cells, the ground-state spin is $S_{g}=N_{c} S_{c}=$ $N_{c}\left|n_{A}-n_{B}\right| S$, and the enhancement ratio equals $K=$ $\left|n_{A}-n_{B}\right|\left(N_{c}\left|n_{A}-n_{B}\right| S+1\right) /\left[\left(n_{A}+n_{B}\right)(S+1)\right]$. It is clear that for a sufficiently large number of cells $N_{c}$ the ratio $K$ will be not only greater than 1 , but can reach very large values. Fig. 3 a shows a honeycomb superstructure that may be formed by TMIs in the $a b$ plane of the wurtzite structure. The hexagon edge length is $a_{h}=2 a$, $a$ being the lattice parameter of the wurtzite. It is easy to imagine superstructures with $a_{h}=2 L a, L=1,2 \ldots$, all of them being ferrimagnetic.

Flat superstructures like those shown in Fig. 3 a can be linked together by some bridging spins to form a threedimensional ferrimagnetic superstructure, which will un- dergo a ferrimagnetic phase transition, provided that the number of cells is macroscopically large. Figure $3 \mathrm{~b}, \mathrm{c}$
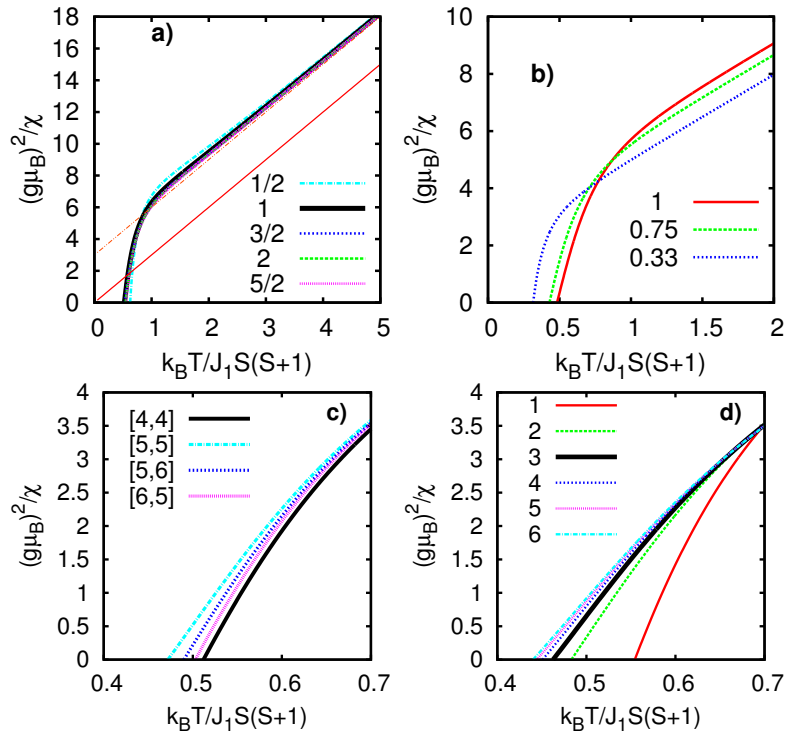

FIG. 4. (Color online) Temperature dependence of inverse susceptibility given by [5,5] Padé approximants for tenthorder high-temperature expansion (HTE) for ferrimagnetic superstructures: a) two-leg honeycomb $(L=1)$, various spin values are shown, solid (dash-dotted) straight red line shows Curie (Curie-Weiss) law; b) four-leg honeycomb $(L=2), S=5 / 2$ various $J_{2} / J_{1}$ values are shown; c) four-leg system, $L=2, S=2$ various Padé approximants for eighthorder $([4,4])^{23 \mid 24}$, tenth-order $([4,6],[5,5],[6,4])^{25]}$, and elevenorder $\left.([5,6],[6,5])^{\sqrt{26}} \mathrm{HTE} ; \mathbf{d}\right)$ the vicinity of $T_{C}$ for various honeycomb superstructures with size parameter $L=1,2 \ldots 6$, $S=5 / 2, J_{2}=J_{1}$.

shows examples of the structures. It is clear that this motif may be repeated in an infinite number of variations. Like the host wurtzite lattice, the unit cell of the superstructure contains metal ions in two planes. The magnetic ions in one plane (green "down" and brown "up" arrows) form a honeycomb lattice with the hexagon edge $2 L a$. In the second plane, the magnetic ions (gold "up" arrows) occupy the positions nearest to the green "down" arrows. The interaction between the ions in the first plane is $J_{1}$, whereas the interaction between the ions in two adjacent planes is $J_{2}$. We note that the complexes shown in Fig. 1 1 , c are building blocks of the honeycombs. It will be demonstrated below that many other Lieb-Mattis networks can be built of such blocks. The number of magnetic ions in the unit cell is $n_{A}+n_{B}=9 L-1$, the ground state spin of the cell being $S_{c}=S\left|n_{A}-n_{B}\right|=S(3 L-1)$. Now the total number of ions in the cell is $n_{c}=24 L^{2}$. Thus, the concentration of magnetic ions equals $x=(9 L-1) /\left(24 L^{2}\right)$, and can be made very small for sufficiently large $L$. At the same time, the average ground-state spin per magnetic ion, $\left\langle S_{\mathbf{R}}\right\rangle=S_{c} /\left(n_{A}+n_{B}\right)=S(3 L-1) /(9 L-1)$, tends to a finite value, $S / 3$, as $L \rightarrow \infty$.

The inverse magnetic susceptibility $\chi^{-1}$ of such super- 
structures is presented in Fig. 4 as a function of normalized temperature $T / T_{s}$. It was calculated using a program $^{25}$ based on the tenth-order high-temperature expansion (HTE) 27. The program computes the exact coefficients of the HTE as well as its Padé approximants (ratios of two polynomials), $\chi(T) \approx[m, n]=P_{m}(T) / P_{n}(T)$. The Padé approximants allow to extend the region of validity of the HTE down to $T \sim 0.5 T_{s}^{25}$ (Fig. 4 c). This extension sometimes fails if an approximant has a pole in the temperature region of interest. Our experience shows that the $[5,5]$ approximant works well in almost all cases. Sometimes difficulties arise for $S=1 / 2$, and for small $J_{2} / J_{1}$ ratios, i.e., for the extreme quantum case. Nevertheless, due to the weak dependence of the shape of the curve $\chi^{-1}\left(T / T_{s}\right)$ on the spin value $S$ (Fig. 4 a), it can still be analyzed. At $T \gtrsim 3 T_{s}$, the inverse susceptibility follows the Curie-Weiss asymptotic law with $\theta=-\left[S(S+1) / 3 k_{B}\right] 12 L\left(J_{1}+J_{2}\right) /(9 L-1)$. For $T \lesssim T_{s}$ it sharply deviates from the asymptotic behavior and changes sign at $T=T_{C}$. This is the temperature of ferrimagnetic ordering - the Curie temperature.

The precision of the determination of critical temperatures from the zero of $\chi^{-1}$ (Fig. 4. ) was estimated to be about $10 \% 25$. Figure $4 \mathrm{~b}$ shows that $T_{C}$ decreases as the ratio of out-of-plane to in-plane couplings, $J_{2} / J_{1}$, is reduced. At $J_{2}=0$ the system becomes a stack of noninteracting two-dimensional planes, and $T_{C}$ should vanish. This limit lies outside the range of applicability of the HTE, and we postpone its study to future works. Here we mention only that magnetic anisotropy, which is neglected in our study, should act in the opposite direction, i.e., it should enhance the $T_{C}$ as it depresses spin fluctuations.

Figure $4 \mathrm{~d}$ shows that the ordering temperature decreases very slowly as $L$ is increased. Note that the superstructure parameter values $L=1,2,3,4,5,6$ correspond to the following concentrations of magnetic ions: $x=0.33,0.18,0.12,0.09,0.07,0.06$. To get a closer relation to experiments, we may consider, e.g., $\mathrm{ZnO}: \mathrm{Mn}, \mathrm{Co}$, where the in-plain superexchange values are $J_{1} / k_{B} \sim$ $50 \mathrm{~K}^{11|13| 14}$ and $T_{s}=J_{1} S(S+1) / k_{B} \sim 438(188) \mathrm{K}$ for $S=5 / 2(3 / 2)$. For other Co-doped semiconductors $66 \mathrm{~K}$ $\lesssim J_{1} / k_{B} \lesssim 100 \mathrm{~K}^{12|17| 28}$ (and references therein), i.e., $T_{s}$ lies within the interval $248 \mathrm{~K} \lesssim T_{s} \lesssim 375 \mathrm{~K}$. The Mndoped semiconductors have $12 \mathrm{~K} \lesssim \widetilde{J}_{1} / k_{B} \lesssim 32 \mathrm{~K}^{12 \mid 29}$, and $105 \mathrm{~K} \lesssim T_{s} \lesssim 280 \mathrm{~K}$.

Thus, a very diluted system may have an appreciable ordering temperature $\left(T_{C} \gtrsim 100 \mathrm{~K}\right)$ provided that the magnetic ions are arranged in a Lieb-Mattis ferrimagnetic superstructure.

In many aspects, the behavior of a ferrimagnet in its ordered state is similar to that of a ferromagnet with the same value of spontaneous magnetization $M_{s}$. But in a high magnetic field the ferrimagnet exhibits a transition accompanied by reorientation of its sublattices 30.32 . At $T=0$ the magnetization per spin has a constant value, $\mu_{M, s}=g \mu_{B} S\left|n_{A}-n_{B}\right| /\left(n_{A}+n_{B}\right)$, up to a certain critical field, $H_{c, 1}$; then it grows up linearly to the saturation value, $\mu_{M, \max }=g \mu_{B} S$, which is reached at a second critical field, $H_{c, 2}$. For a two-sublattice ferrimagnet having the structure shown in Fig. 3 a $(L=1)$ and $J_{1}=J_{2}=J$ we find $g \mu_{B} H_{c, 1}=J S$, and $H_{c, 2}=5 H_{c, 1}$. For $J / k_{B} \sim 20 \mathrm{~K}$ this gives $H_{c, 1} \sim 37 \mathrm{~T}, H_{c, 2} \sim 185 \mathrm{~T}$.

The complexes shown in Fig. 1 b,c may be arranged in many kinds of networks, to form Lieb-Mattis ferrimagnetic superstructures in various host semiconductors. Figure $3 \mathrm{~d}$ shows an example of a 2D square superstructure unit cell with $L=2$, which is possible in a cubic host. It has $n_{A}=1+2(L-1)$ and $n_{B}=4(L-1)+2 L$. One can also imagine a $3 \mathrm{D}$ cubic network; then Fig. $3 \mathrm{~d}$ corresponds to a face of the cubic unit cell having $n_{A}=$ $1+3(L-1), n_{B}=3 L+12(L-1)$, and the concentration of magnetic ions $x=\left(n_{A}+n_{B}\right) / n_{c}=(9 L-7) /\left(4 L^{3}\right)$. Formation of such superstructures is possible in perovskite solid solutions, like $\mathrm{KMn}_{x} \mathrm{Mg}_{1-x} \mathrm{~F}_{3}$ 33/34, or in solutions of multiferroics $\mathrm{PbFe}_{1 / 2} \mathrm{Nb}_{1 / 2} \mathrm{O}_{3}$ or $\mathrm{PbFe}_{1 / 2} \mathrm{Ta}_{1 / 2} \mathrm{O}_{3}$ with ferroelectric perovskites 35 .

We conclude that Lieb-Mattis ferrimagnetism is a possible route to obtaining long-range magnetic order in semiconductors containing transition metal ions as substitutional impurities, which requires no additional charge carriers. A precursor of the ordering transition is the enhanced magnetic response of finite cluster showing isotropic superparamagnetism. Our results for the inverse susceptibility show a characteristic " $\mathrm{S}$ "-like form of the curves, which could be used to identify the present mechanism. Adding the magnetic anisotropy to our theory, we expect also other ingredients of superparamagnetism, namely a finite blocking temperature and hysteresis.

These superparamagnetic clusters serve as building blocks to create infinite sublattices of the wurtzite structure that obey the Lieb-Mattis rules. As we have already noted, there is an enormous wealth of such LiebMattis sublattices, our proposals (Fig. 3) may only serve as examples. We expect a finite transition temperature for all these lattices and we have shown it explicitly for the subclass that we considered. Of course, a question arises, whether frustration in a realistic diluted semiconductor can influence the above discussed scenario. First we argue that there are several numerical studies showing that the Lieb-Mattis theorem, although not rigorously valid, applies to many frustrated spin systems, see, e.g., Ref. 40, Furthermore, we know that there are various frustrated $2 \mathrm{D}$ lattices with antiferromagnetic nearest-neighbor exchange, such as the triangular or the Shastry-Sutherland lattices, which show ground-state magnetic LRO ${ }^{4142}$. Last but not least, the stability of the ferrimagnetic ground state against frustration has been demonstrated for several specific ferrimagnetic models, see, e.g., Refs. 43 45. Consequently, there is ample evidence that the above sketched mechanism should be robust against frustration. The final proof that the here proposed mechanism can, indeed, be realized in a real material demands further studies, in close collaboration between experiment and theory. 
In this communication, we have considered only semiconductors doped by one kind of magnetic ions, where ferrimagnetism can appear due to the topology of interacting bonds. Another option is the co-doping with two kinds of ions having different spin values. In both cases a ferrimagnetic semiconductor may be a good alternative to a ferromagnetic one.

\section{ACKNOWLEDGMENTS}

The projects NASc of Ukraine 07-02-15, and NATO project SfP 984735 are acknowledged. The exact diagonalization calculations were performed using J. Schulenburg's spinpack.
1 I. Žutić, J. Fabian, and S. Das Sarma, Rev. Mod. Phys. 76, 323 (2004), URL http://link.aps.org/doi/ 10.1103/RevModPhys.76.323.

${ }^{2}$ F. Matsukura, H. Ohno, A. Shen, and Y. Sugawara, Phys. Rev. B 57, R2037 (1998).

3 T. Dietl, H. Ohno, F. Matsukura, J. Cibert, and D. Ferrand, Science 287, 1019 (2000).

${ }^{4}$ C. Zener, Phys. Rev. 82, 403 (1951).

5 T. Dietl, Nat. Mater. 9, 965 (2010), URL http://dx.doi. org/10.1038/nmat2898

${ }^{6}$ R. Janisch, P. Gopal, and N. A. Spaldin, Journal of Physics: Condensed Matter 17, R657 (2005), URL http: //stacks. iop.org/0953-8984/17/i=27/a=R01.

S. B. Ogale, Advanced Materials 22, 3125 (2010), ISSN 1521-4095, URL http://dx.doi.org/10.1002/ adma. 200903891.

8 E. Lieb and D. Mattis, Journal of Mathematical Physics 3, 749 (1962), URL http://link.aip.org/link/?JMP/3/ $749 / 1$.

${ }^{9}$ S. Bedanta and W. Kleemann, Journal of Physics D: Applied Physics 42, 013001 (2009), URL http://stacks. iop.org/0022-3727/42/i=1/a=013001.

I0 R. O. Kuzian, V. V. Laguta, and J. Richter, Phys. Rev. B 90, 134415 (2014), URL http://link.aps.org/doi/10. 1103/PhysRevB.90.134415

11 In the literature one meets also the notation $-2 J_{\mathbf{r}, L}$ for the same exchange parameter.

12 Y. Shapira and V. Bindilatti, Journal of Applied Physics 92, 4155 (2002), URL http://scitation.aip.org/ content/aip/journal/jap/92/8/10.1063/1.1507808.

${ }^{13}$ X. Gratens, V. Bindilatti, N. F. Oliveira, Y. Shapira, S. Foner, Z. Golacki, and T. E. Haas, Phys. Rev. B 69, 125209 (2004), URL http://link.aps .org/doi/10.1103/ PhysRevB.69.125209

${ }^{14}$ S. D'Ambrosio, V. Pashchenko, J.-M. Mignot, O. Ignatchik, R. O. Kuzian, A. Savoyant, Z. Golacki, K. Grasza, and A. Stepanov, Phys. Rev. B 86, 035202 (2012), URL http://link.aps.org/doi/10.1103/PhysRevB.86. 035202

15 T. Chanier, M. Sargolzaei, I. Opahle, R. Hayn, and K. Koepernik, Phys. Rev. B 73, 134418 (2006).

16 R. O. Kuzian, A. M. Daré, A. Savoyant, S. D'Ambrosio, and A. Stepanov, Phys. Rev. B 84, 165207 (2011), URL http://link.aps.org/doi/10.1103/PhysRevB.84. 165207

17 A. Savoyant, S. D'Ambrosio, R. O. Kuzian, A. M. Daré, and A. Stepanov, Phys. Rev. B 90, 075205 (2014), URL http://link.aps.org/doi/10.1103/PhysRevB.90. 075205

${ }^{18}$ M. T. Liu, Y. Shapira, E. ter Haar, V. Bindilatti, and
E. J. McNiff, Phys. Rev. B 54, 6457 (1996), URL http: //link.aps.org/doi/10.1103/PhysRevB.54.6457

19 The term "ferri-magnetism" for finite system means that, on one hand, quantum mechanical average over the ground state of operators of neighboring spins $\left\langle\hat{S}_{\mathbf{R}} \hat{S}_{\mathbf{R}+\rho}\right\rangle$ (vector $\rho$ connect neighboring spin positions)is negative (in average, the neighboring spins are aligned in opposite directions), whereas, on the other hand, the total ground state spin of the system $S_{g}$ is non-zero.

20 spinpack is available at http://www-e.unimagdeburg.de/jschulen/spin/.

21 J. Richter and J. Schulenburg, Eur. Phys. J. B 73, 117 (2010).

${ }^{22}$ V. V. Laguta, V. A. Stephanovich, M. Savinov, M. Marysko, R. O. Kuzian, I. V. Kondakova, N. M. Olekhnovich, A. V. Pushkarev, Y. V. Radyush, I. P. Raevski, et al., New Journal of Physics 16, 113041 (2014), URL http://stacks.iop.org/1367-2630/16/i= $11 / \mathrm{a}=113041$

${ }^{23}$ H.-J. Schmidt, A. Lohmann, and J. Richter, Phys. Rev. B 84, 104443 (2011), URL http://link.aps.org/doi/10. 1103/PhysRevB.84.104443

${ }^{24}$ For eight-order HTE, we have used the 201109-23 version of HTE package available at http://www.uni-magdeburg.de/jschulen/HTE/, URL http://www .uni-magdeburg.de/jschulen/HTE/

25 A. Lohmann, H.-J. Schmidt, and J. Richter, Phys. Rev. B 89, 014415 (2014), URL http://link.aps.org/doi/10. 1103/PhysRevB.89.014415

${ }^{26}$ We thank A. Lohmann for providing the code of the 11th order HTE.

27 For tenth-order HTE, we have used HTE10 package available at http://www.uni-magdeburg.de/jschulen/HTE10/, URL http://www . uni-magdeburg.de/jschulen/HTE10/

28 T. M. Giebultowicz, J. J. Rhyne, J. K. Furdyna, and P. Klosowski, Journal of Applied Physics 67, 5096 (1990), URL http://scitation.aip.org/content/aip/ journal/jap/67/9/10.1063/1.344683.

29 S. Foner, Y. Shapira, D. Heiman, P. Becla, R. Kershaw, K. Dwight, and A. Wold, Phys. Rev. B 39, 11793 (1989), URL http://link.aps.org/doi/10.1103/ PhysRevB.39.11793

so S. V. Tyablikov, Fiz. Metallov. i Metallovedenie 3, 3 (1956).

S. V. Tyablikov, Methods in the Quantum Theory of Magnetism (Plenum, New York, 1967).

31 E. Schlömann, in Solid State Physics in Electronics and Telecommunications, edited by M. Désirant and J. Michiels (Academic Press, London, 1960).

32 A. E. Clark and E. Callen, Journal of Applied Physics 39, 
5972 (1968), URL http://scitation.aip.org/content/ aip/journal/jap/39/13/10.1063/1.1656100

33 G. D'Ariano and F. Borsa, Phys. Rev. B 26, 6215 (1982), URL http://link.aps.org/doi/10.1103/PhysRevB.26. 6215 .

${ }^{34}$ D. J. Breed, K. Gilijamse, J. W. E. Sterkenburg, and A. R. Miedema, J. Appl. Phys. 41, 1267 (1970), URL http:// dx.doi.org.sci-hub.org/10.1063/1.1658906

35 D. A. Sanchez, N. Ortega, A. Kumar, G. Sreenivasulu, R. S. Katiyar, J. F. Scott, D. M. Evans, M. ArredondoArechavala, A. Schilling, and J. M. Gregg, Journal of Applied Physics 113, 074105 (2013), URL http://link . aip. org/link/?JAP/113/074105/1

${ }^{36}$ D. Evans, A. Schilling, A. Kumar, D. Sanchez, N. Ortega, M. Arredondo, R. Katiyar, J. Gregg, and J. Scott, Nat. Commun. 4, 1534 (2013), URL http://dx.doi.org/10. $1038 /$ ncomms 2548

37 V. V. Laguta, M. D. Glinchuk, M. Maryško, R. O. Kuzian, S. A. Prosandeev, S. I. Raevskaya, V. G. Smotrakov, V. V. Eremkin, and I. P. Raevski, Phys. Rev. B 87, 064403 (2013), URL http://link.aps .org/doi/10.1103/ PhysRevB.87.064403
38 D. A. Sanchez, N. Ortega, A. Kumar, R. Roque-Malherbe, R. Polanco, J. F. Scott, and R. S. Katiyar, AIP Advances 1, 042169 (2011), URL http://link.aip.org/link/?ADV/ $1 / 042169 / 1$.

39 A. Kumar, G. L. Sharma, R. S. Katiyar, R. Pirc, R. Blinc, and J. F. Scott, Journal of Physics: Condensed Matter 21, 382204 (2009), URL http://stacks .iop.org/0953-8984/ $21 / i=38 / a=382204$

40 J. Richter, N. Ivanov, K. Retzlaff, and A. Voigt, J. Magn. Magn. Mat. 140-144, 1611 (1995).

41 J. Richter, J. Schulenburg, and A. Honecker, Lect. Notes Phys. 645, 85 (2004).

42 D.J.J. Farnell, O. Götze, J. Richter, R.F. Bishop, and P.H.Y. Li, Phys. Rev. B 89, 184407 (2014).

43 N.B. Ivanov, J. Richter, and U. Schollwöck, Phys. Rev. B 58, 14456 (1998).

44 C. Waldtmann, H. Kreutzmann, U. Schollwöck, K. Maisinger, and H.-U. Everts, Phys. Rev. B 62, 9472 (2000).

45 N.B. Ivanov, J. Richter, and D.J.J. Farnell, Phys. Rev. B 66, 014421 (2002). 